\title{
On a Class of Submanifolds in a Tangent Bundle with a $g$-Natural Metric - Normal lift
}

\author{
Stanisław Ewert-Krzemieniewski*
}

(Communicated by Murat Tosun)

\begin{abstract}
An isometric immersion of a Riemannian manifold $M$ into a Riemannian manifold $N$ gives rise in a natural way to variety of immersions into the tangent bundle $T N$ with a non-degenerate $g$-natural metric $G$. In the paper we introduce and study an immersion into $T N$ defined by the immersion $f: M \longrightarrow N$ itself and the normal bundle.
\end{abstract}

Keywords: Riemannian manifold; tangent bundle; g-natural metric; submanifold; isometric immersion; totally geodesic distribution; non-degenerate metric. AMS Subject Classification (2010): Primary: 53B20; 53C07; Secondary: 53B21; 55C25.

\section{Introduction}

An isometric immersion of a Riemannian manifold $M$ into a Riemannian manifold $N$ gives rise in a natural way to variety of immersions into the tangent bundle $T N$ with a non-degenerate $g$-natural metric $G$. The isometric immersion defined by the tangent bundle of the submanifold was introduced by the author in [11], [12]. In the present paper we introduce and study an immersion $\tilde{f}: L M \longrightarrow T N$ defined by the immersion $f: M \longrightarrow N$ itself and the normal bundle.

In Preliminaries we recall basic facts on the decomposition of the tangent bundle and $g$-natural metrics. We also present basic notions on submanifolds and give short resumé on van der Waerden-Bortolotti covariant derivative. In Section 3 basic equations are presented. The main results are given in Section 4 . We give the condition sufficient for $L M$ being totally geodesic submanifold of $T N$.

Throughout the paper all manifolds under consideration are Hausdorff and smooth. The metrics on the base manifolds are Riemannian and the metrics on tangent spaces are non-degenerate. We adopt the Einstein summation convention.

\section{Preliminaries}

\subsection{Decomposition of the tangent space}

Let $\pi: T N \longrightarrow N$ be the tangent bundle of a Riemannian manifold $N$ with the Levi-Civita connection $\nabla$ on $N, \pi$ being the projection. Then at each point $(x, u) \in T N$ the tangent space $T_{(x, u)} T N$ splits into direct sum of two isomorphic spaces $V_{(x, u)} T N$ and $H_{(x, u)} T N$, where

$$
V_{(x, u)} T N=\operatorname{Ker}\left(\left.d \pi\right|_{(x, u)}\right), \quad H_{(x, u)} T N=\operatorname{Ker}\left(\left.K\right|_{(x, u)}\right)
$$

and $K$ is the connection map [7], see also [15].

More precisely, if $Z=\left.\left(Z^{r} \frac{\partial}{\partial x^{r}}+\bar{Z}^{r} \frac{\partial}{\partial u^{r}}\right)\right|_{(x, u)} \in T_{(x, u)} T N, r=1, \ldots, n$, then the vertical and horizontal projections of $Z$ on $T_{x} N$ are given by

$$
(d \pi)_{(x, u)} Z=\left.Z^{r} \frac{\partial}{\partial x^{r}}\right|_{x}, \quad K_{(x, u)}(Z)=\left.\left(\bar{Z}^{r}+u^{s} Z^{t} \Gamma_{s t}^{r}\right) \frac{\partial}{\partial x^{r}}\right|_{x}
$$


where $\Gamma_{s t}^{r}$ are components of the Levi-Civita connection on $N$.

On the other hand, to each vector field $X$ on $N$ there correspond uniquely determined vector fields $X^{v}$ and $X^{h}$ on $T N$ such that

$$
\begin{aligned}
\left.d \pi\right|_{(x, u)}\left(X^{v}\right) & =0,\left.\quad K\right|_{(x, u)}\left(X^{v}\right)=X, \\
\left.K\right|_{(x, u)}\left(X^{h}\right) & =0,\left.\quad d \pi\right|_{(x, u)}\left(X^{h}\right)=X .
\end{aligned}
$$

$X^{v}$ and $X^{h}$ are called the vertical lift and the horizontal lift of a given $X$ to $T N$, respectively.

In local coordinates $\left(\left(x^{r}\right),\left(u^{r}\right)\right), r=1, . ., n$, on $T N$, the horizontal and vertical lifts of a vector field $X=X^{r} \frac{\partial}{\partial x^{r}}$ on $N$ to $T N$ are vector fields given respectively by

$$
X^{h}=X^{r} \frac{\partial}{\partial x^{r}}-u^{s} X^{t} \Gamma_{s t}^{r} \frac{\partial}{\partial u^{r}}, \quad X^{v}=X^{r} \frac{\partial}{\partial u^{r}} .
$$

Recall that for a given isometric immersion $f: M \longrightarrow N$, we have two tangent bundles $\pi_{N}: T N \longrightarrow N$ and $\pi_{M}: T M \longrightarrow M$, where the latter is the subbundle of the former. Let $M, N$ be two Riemannian manifolds with metrics $g_{M}$ and $g_{N}$ and Levi-Civita connections $\nabla_{M}$ and $\nabla_{N}$ respectively. Then $T_{p} T M$ and $T_{p} T N$ have at a common point $p$ their own decompositions into vertical and horizontal parts, i.e.

$$
T_{p} T M=V_{p} T M \oplus H_{p} T M=V_{M} \oplus H_{M}
$$

and

$$
T_{p} T N=V_{p} T N \oplus H_{p} T N=V_{N} \oplus H_{N},
$$

but neither $V_{M} \subset V_{N}$ nor $H_{M} \subset H_{N}$ need to hold along $T M$. See also [15]

Remark also that totally geodesic submanifolds of tangent bundle with $g$-natural metric are also studied in [1] and [10].

\subsection{Preliminaries on g-natural metrics}

In [13] the class of $g$-natural metrics was defined. We have

Lemma 2.1. ([13], [2], [3]) Let $(M, g)$ be a Riemannian manifold and $G$ be a g-natural metric on TM. There exist functions $\left.a_{j}, b_{j}:<0, \infty\right) \longrightarrow R, j=1,2,3$, such that for every $X, Y, u \in T_{x} M$

$$
\begin{array}{r}
G_{(x, u)}\left(X^{h}, Y^{h}\right)=\left(a_{1}+a_{3}\right)\left(r^{2}\right) g_{x}(X, Y)+\left(b_{1}+b_{3}\right)\left(r^{2}\right) g_{x}(X, u) g_{x}(Y, u), \\
G_{(x, u)}\left(X^{h}, Y^{v}\right)=G_{(x, u)}\left(X^{v}, Y^{h}\right)=a_{2}\left(r^{2}\right) g_{x}(X, Y)+b_{2}\left(r^{2}\right) g_{x}(X, u) g_{x}(Y, u), \\
\quad G_{(x, u)}\left(X^{v}, Y^{v}\right)=a_{1}\left(r^{2}\right) g_{x}(X, Y)+b_{1}\left(r^{2}\right) g_{x}(X, u) g_{x}(Y, u),
\end{array}
$$

where $r^{2}=g_{x}(u, u)$. For $\operatorname{dim} M=1$ the same holds for $b_{j}=0, j=1,2,3$.

Setting $a_{1}=1, a_{2}=a_{3}=b_{j}=0$ we obtain the Sasaki metric, while setting $a_{1}=b_{1}=\frac{1}{1+r^{2}}, a_{2}=b_{2}=0=0$, $a_{1}+a_{3}=1, b_{1}+b_{3}=1$ we get the Cheeger-Gromoll one.

Following [2] we put

1. $a(t)=a_{1}(t)\left(a_{1}(t)+a_{3}(t)\right)-a_{2}^{2}(t)$,

2. $F_{j}(t)=a_{j}(t)+t b_{j}(t)$,

3. $F(t)=F_{1}(t)\left[F_{1}(t)+F_{3}(t)\right]-F_{2}^{2}(t)$

for all $t \in<0, \infty)$.

We shall often abbreviate: $A=a_{1}+a_{3}, B=b_{1}+b_{3}$.

Lemma 2.2. ([2], Proposition 2.7) The necessary and sufficient conditions for a g-natural metric $G$ on the tangent bundle of a Riemannian manifold $(M, g)$ to be non-degenerate are

$$
a(t) \neq 0, \quad F(t) \neq 0
$$

for all $t \in<0, \infty)$. If $\operatorname{dim} M=1$, this is equivalent to $a(t) \neq 0$ for all $t \in<0, \infty)$.

Moreover, $(T M, G)$ is Riemannian one if and only if

$$
a(t)>0, \quad F(t)>0, \quad a_{1}(t)>0, \quad F_{1}(t)>0
$$

hold for all $t \in<0, \infty)$. 
We also have

Proposition 2.1. ([4], [5]) Let $(N, g)$ be a Riemannian manifold, $\nabla$ its Levi-Civita connection and $R$ its Riemann curvature tensor. If $G$ is a non-degenerate g-natural metric on $T N$, then the Levi-Civita connection $\widetilde{\nabla}$ of $(T N, G)$ is given at a point $(x, u) \in T N$ by

$$
\begin{gathered}
\left(\widetilde{\nabla}_{X^{h}} Y^{h}\right)_{(x, u)}=\left(\nabla_{X} Y\right)_{(x, u)}^{h}+h\left\{\mathbf{A}\left(u, X_{x}, Y_{x}\right)\right\}+v\left\{\mathbf{B}\left(u, X_{x}, Y_{x}\right)\right\}, \\
\left(\widetilde{\nabla}_{X^{h}} Y^{v}\right)_{(x, u)}=\left(\nabla_{X} Y\right)_{(x, u)}^{v}+h\left\{\mathbf{C}\left(u, X_{x}, Y_{x}\right)\right\}+v\left\{\mathbf{D}\left(u, X_{x}, Y_{x}\right)\right\}, \\
\left(\widetilde{\nabla}_{X^{v}} Y^{h}\right)_{(x, u)}=h\left\{\mathbf{C}\left(u, Y_{x}, X_{x}\right)\right\}+v\left\{\mathbf{D}\left(u, Y_{x}, X_{x}\right)\right\}, \\
\left(\widetilde{\nabla}_{X^{v}} Y^{v}\right)_{(x, u)}=h\left\{\mathbf{E}\left(u, X_{x}, Y_{x}\right)\right\}+v\left\{\mathbf{F}\left(u, X_{x}, Y_{x}\right)\right\},
\end{gathered}
$$

where $\mathbf{A}, \mathbf{B}, \mathbf{C}, \mathbf{D}, \mathbf{E}, \mathbf{F}$ are some F-tensors defined on the product $T N \otimes T N \otimes T N$.

Remark 2.1. Expressions for A, B, C, D, E, F were presented for the first time in the original papers ([2], [3]). Unfortunately, they contain some misprints and omissions. Therefore, for the correct form, we refer the reader to $([4],[5])$, see also ([8], [9]).

\subsection{Submanifolds}

Let $M$ be a manifold isometrically immersed in a pseudo-Riemannian manifold $N$ with metric $g$. Denote by $\widetilde{\nabla}$ and $\nabla$ the Levi-Civita connections of the metric $g$ on $N$ and that of the induced metric on $M$ and by $D^{\perp}$ the connection induced in the normal bundle $T^{\perp} M$. Then the Gauss and Weingarten equations

$$
\begin{gathered}
\widetilde{\nabla}_{X} Y=\nabla_{X} Y+H(X, Y), \\
\widetilde{\nabla}_{X} \eta=-A_{\eta} X+D_{X}^{\perp} Y,
\end{gathered}
$$

hold for all vectors fields $X, Y$ tangent to $M$ and all vector fields $\eta$ normal to $M$. Here $H(X, Y)$ is the second fundamental form which is symmetric and takes values in $T^{\perp} M$ while $A_{\eta} X$ is the shape operator taking values in $T M$. It is well known that $A_{\eta}$ and $H$ are related by

$$
g\left(A_{\eta} X, Y\right)=g(\eta, H(X, Y)) .
$$

$M$ is said to be totally geodesic if $H(X, Y)=0$ for all $X, Y \in T M$.

For the local immersion $x^{r}=x^{r}\left(y^{a}\right), r=1, \ldots, n, a=1, \ldots, m$, the components of the Levi-Civita connection $\nabla$ of the induced metric $g_{a b}=g_{r s} B_{a}^{r} B_{b}^{s}, B_{a}^{r}=\frac{\partial x^{r}}{\partial y^{a}}$, are

$$
\Gamma_{a b}^{c}=\left[B_{a . b}^{r}+\Gamma_{s t}^{r} B_{a}^{s} B_{b}^{t}\right] B_{r}^{c}, \quad B_{r}^{c}=g^{c d} B_{d}^{t} g_{t r},
$$

where the dot denotes partial derivative with respect to $y^{b}$.

Similarly, the components of the connection $D^{\perp}$ are

$$
\Gamma_{a y}^{x}=\left[N_{y \cdot a}^{r}+\Gamma_{s t}^{r} B_{a}^{s} N_{y}^{t}\right] N_{r}^{x}, \quad N_{r}^{x}=g^{x y} N_{y}^{t} g_{t r},
$$

where $\eta_{z}=N_{z}^{r} \frac{\partial}{\partial x^{r}}, z=m+1, \ldots, n$ are unit vector fields normal to $M$.

2.3.1. Van der Waerden-Bortolotti covariant derivative Van der Waerden-Bortolotti covariant derivative $\bar{\nabla}$ is a covariant differentiation of tensor fields of mixed types defined along a submanifold $M$ isometrically immersed in a pseudo-Riemannian manifold $(N, g)$ and can be considered as a direct sum $\widetilde{\nabla} \oplus \nabla \oplus \nabla^{\perp}$ of the Levi-Civita connections of the metric $g$ on $N$, the one induced on $M$ and of the metric induced in normal bundle $T^{\perp} M$. 
If $\widetilde{X}, X$ and $\eta$ are vector fields, respectively, tangent to $N$, tangent to $M$, normal to $M$ and $\widetilde{X}^{*}, X^{*}, \eta^{*}$ are respective 1 -forms, then,

$$
\begin{aligned}
&\left(\bar{\nabla}_{Y} T\right)\left(\widetilde{X}, X, \eta, \widetilde{X}^{*}, X^{*}, \eta^{*}\right)=Y\left(T\left(\widetilde{X}, X, \eta, \widetilde{X}^{*}, X^{*}, \eta^{*}\right)\right)- \\
& T\left(\widetilde{\nabla} Y \widetilde{X}, X, \eta, \widetilde{X}^{*}, X^{*}, \eta^{*}\right)-T\left(\widetilde{X}, X, \eta, \widetilde{\nabla}_{Y} \widetilde{X}^{*}, X^{*}, \eta^{*}\right)- \\
& T\left(\widetilde{X}, \nabla_{Y} X, \eta, \widetilde{X}^{*}, X^{*}, \eta^{*}\right)-T\left(\widetilde{X}, X, \eta, \widetilde{X}^{*}, \nabla_{Y} X^{*}, \eta^{*}\right)- \\
& T\left(\widetilde{X}, X, \nabla_{Y}^{\perp} \eta, \widetilde{X}^{*}, X^{*}, \eta^{*}\right)-T\left(\widetilde{X}, X, \eta, \widetilde{X}^{*}, X^{*}, \nabla_{Y}^{\perp} \eta^{*}\right)
\end{aligned}
$$

for any vector field $Y$ tangent to $M$ and tensor field $T$ of mixed type $(3,3)$.

Let $x^{k}=x^{k}\left(y^{a}\right)$ be the local expression of the immersion, $B_{a}^{k}=\frac{\partial x^{k}}{\partial y^{a}}$. Let $\eta_{x}=N_{x}^{r} \frac{\partial}{\partial x^{r}}, x=m+1, \ldots, n$, be an orthonormal set of vectors normal to $M$.

For the local coordinate vector fields $\frac{\partial}{\partial x^{k}}$ tangent to $N, \frac{\partial}{\partial y^{a}}$ tangent to $M, \frac{\partial}{\partial v^{x}}$ normal to $M$ and the respective 1 -forms $d x^{k}, d y^{a}, d v^{x}$, denote by $\Gamma_{h k}^{l}, \Gamma_{a b}^{c}, \Gamma_{a y}^{z}$ components of the connections $\widetilde{\nabla}, \nabla$ and $\nabla^{\perp}$. If

$$
T=T_{h a x}^{k b y} \frac{\partial}{\partial x^{k}} \otimes d x^{h} \otimes \frac{\partial}{\partial y^{b}} \otimes d y^{a} \otimes \frac{\partial}{\partial v^{y}} \otimes d v^{x},
$$

then

$$
\nabla_{c} T_{h a x}^{k b y}=\partial_{b} T_{h a x}^{k b y}-\Gamma_{h r}^{s} B_{c}^{r} T_{s a x}^{k b y}+\Gamma_{r s}^{k} B_{c}^{r} T_{h a x}^{s b y}-
$$

$$
\Gamma_{c a}^{d} T_{h d x}^{k b y}+\Gamma_{c d}^{b} T_{h a x}^{k d y}-\Gamma_{c x}^{z} T_{h a z}^{k b y}+\Gamma_{c z}^{y} T_{h a x}^{k b z},
$$

where $h, k, r, s=1, \ldots, n, a, b, c, d=1, \ldots, m, m<n$, and $x, y, z=m+1, \ldots, n$.

In particular, $\bar{\nabla}_{a} B_{b}^{r}$ and $\bar{\nabla}_{a} N_{x}^{r}$ give rise to the components of the second fundamental form and the shape operator:

$$
\begin{gathered}
\bar{\nabla}_{b} B_{a}^{r} \partial_{r}=\left(B_{a . b}^{r}+\Gamma_{s t}^{r} B_{a}^{s} B_{b}^{t}-\Gamma_{a b}^{c} B_{c}^{r}\right) \partial_{r}=h_{a b}^{z} N_{z}^{r} \partial_{r}, \\
\bar{\nabla}_{a} N_{x}^{r} \partial_{r}=\left(\partial_{a} N_{x}^{r}+\Gamma_{s t}^{r} B_{a}^{s} N_{x}^{t}-\Gamma_{a x}^{y} N_{y}^{r}\right) \partial_{r} .
\end{gathered}
$$

In a free of coordinate notation we have respectively:

$$
\begin{aligned}
& \bar{\nabla}_{X} Y=\widetilde{\nabla}_{X} Y-\nabla_{X} Y, \\
& \bar{\nabla}_{X} \eta=\widetilde{\nabla}_{X} \eta-D_{X}^{\perp} \eta .
\end{aligned}
$$

See also [14] and [16].

2.3.2. Isometric immersion defined by normal bundle Let $f: M \longrightarrow N$ be an isometric immersion of a Riemannian manifold $M$ into a Riemannian manifold $N$. Suppose that the following diagram commute

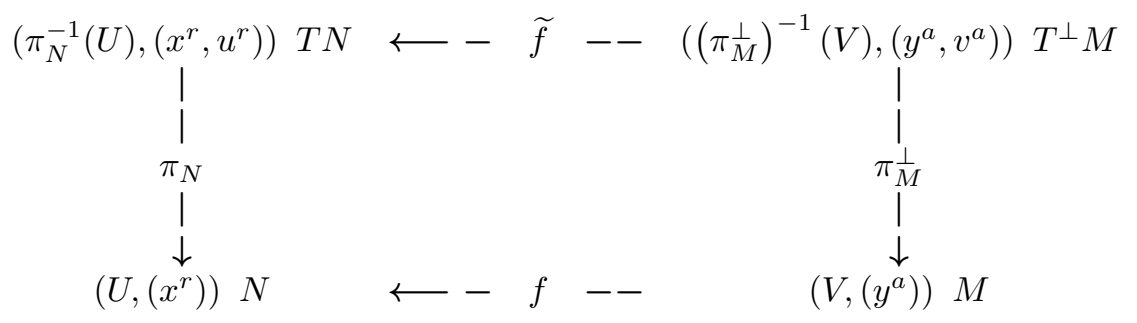

where $\left(U,\left(x^{r}\right)\right)$ and $\left(V,\left(y^{a}\right)\right)$ are coordinate neighbourhoods on $N$ and $M$ respectively, while the local expression for $f$ is:

$$
f: x^{r}=x^{r}\left(y^{a}\right) .
$$

Let

$$
\eta_{x}=N_{x}^{r} \frac{\partial}{\partial x^{r}}, \quad x=m+1, \ldots, n
$$


be a set of orthonormal vectors normal to $M$.

The coordinate neighbourhoods on $T N$ and normal bundle $T^{\perp} M$ are defined respectively by

$$
\begin{gathered}
\left(\left(x^{r}\right),\left(u^{r}\right)\right), \quad r=1, \ldots, n, \\
\left(\left(y^{a}\right),\left(v^{x}\right)\right), \quad x=m+1, \ldots, n, \quad a=1, \ldots, m,
\end{gathered}
$$

where $\left(u^{r}\right)_{r=1, \ldots, n}$, are components of the vector $u$ tangent to $N$ at a point with coordinates $\left(x^{r}\right)$ and $\left(v^{x}\right)_{x=m+1, \ldots, n}$ are components of the vector normal to $M$ at a point $x^{r}=x^{r}\left(y^{a}\right)$.

If

$$
f: M \longrightarrow N ; \quad\left(y^{a}\right) \mapsto x^{r}\left(y^{a}\right)
$$

then

$$
\widetilde{f}: x^{r}=x^{r}\left(y^{a}\right), \quad u^{r}=v^{x} N_{x}^{r}
$$

defines locally an immersion into $T N$.

2.3.3. Vectors tangent to $L M$ The coordinate vector fields tangent to $L M=\widetilde{f}\left(T M^{\perp}\right)$ are

$$
\frac{\partial}{\partial v^{x}}=N_{x}^{r} \frac{\partial}{\partial u^{r}}=\left(\eta_{x}\right)^{v}
$$

$$
\begin{gathered}
\frac{\partial}{\partial y^{a}}=B_{a}^{r} \frac{\partial}{\partial x^{r}}+v^{z} \partial_{a} N_{z}^{r} \frac{\partial}{\partial u^{r}} \stackrel{(2.2)}{=} \\
B_{a}^{r}\left(\frac{\partial}{\partial x^{r}}-v^{z} N_{z}^{t} \Gamma_{t r}^{s} \frac{\partial}{\partial u^{s}}\right)+v^{z} \bar{\nabla}_{a} N_{z}^{r} \frac{\partial}{\partial u^{r}}+v^{z} \Gamma_{a z}^{y} N_{y}^{r} \frac{\partial}{\partial u^{r}} \stackrel{(2.3),(2.1)}{=} \\
\left(\frac{\delta}{\delta y^{a}}\right)^{h}+M_{a}^{r}\left(\frac{\partial}{\partial x^{r}}\right)^{v}+N_{a}^{r}\left(\frac{\partial}{\partial x^{r}}\right)^{v}= \\
\left(\frac{\delta}{\delta y^{a}}\right)^{h}+\left(M_{a}\right)^{v}+\left(N_{a}\right)^{v},
\end{gathered}
$$

where $M_{a}=v^{z} \bar{\nabla}_{a} N_{z}^{r} \frac{\partial}{\partial x^{r}}=v^{z} M_{a z}$ are tangent to $M$ and $N_{a}=v^{z} \Gamma_{a z}^{y} N_{y}^{r} \frac{\partial}{\partial x^{r}}=N_{a}^{y} N_{y}^{r} \frac{\partial}{\partial x^{r}}$ are normal to $M$.

Along $M$ we also have

$$
\nabla_{\delta_{a}} u=\nabla_{\delta_{a}}\left(v^{y} \eta_{y}\right)=\nabla_{\delta_{a}}\left(v^{y} N_{y}^{r} \frac{\partial}{\partial x^{r}}\right)=M_{a}+N_{a} .
$$

\section{Basic equations}

In this section we derive, using the equations of Gauss and Weingarten and the formulas for the Levi-Civita connection on $(T N, G)$ with a non-degenerate $g$-natural metric $G$, the basic equations for the immersion given by (2.3) to be used throughout the paper. $H^{h}+V^{v}$ is a unique decomposition of a vector field normal to $L M$ into its horizontal and vertical parts, where $H$ and $V$ are vector fields along $M$, not necessary tangent to $M$. $\nabla$ and $\widetilde{\nabla}$ denote the Levi-Civita connections of the metric $g$ and $g$-natural non-degenerate metric $G$, respectively. $\widetilde{H}$ is the second fundamental form of the immersion (2.3). Finally, $R$ stands for the Riemann curvature tensor of $g$. The computations in this section were performed and checked with Mathematica software. In virtue of Proposition 4.1, the pairs of equations in each subsection must satisfy

$$
G\left(\widetilde{H}\left(\partial_{x}, \partial_{y}\right), H^{h}+V^{v}\right)-G\left(\widetilde{A}_{H^{h}+V^{v}} \partial_{x}, \partial_{y}\right)=0 .
$$

It also can be used to verify the correctness of computations. 


\section{Equation 1}

$$
\begin{gathered}
G\left(\widetilde{\nabla}_{\partial_{x}} \partial_{y}, H^{h}+V^{v}\right)=G\left(\widetilde{H}\left(\partial_{x}, \partial_{y}\right), H^{h}+V^{v}\right)= \\
G\left(\widetilde{\nabla}_{\eta_{x}^{v}} \eta_{y}^{v}, H^{h}+V^{v}\right)= \\
G\left(h\left\{\mathbf{E}\left(u, \eta_{x}, \eta_{y}\right)\right\}+v\left\{\mathbf{F}\left(u, \eta_{x}, \eta_{y}\right)\right\}, H^{h}+V^{v}\right)=
\end{gathered}
$$

$b_{2} g\left(\eta_{x}, \eta_{y}\right) g(u, H)+\left(b_{1}-a_{1}^{\prime}\right) g\left(\eta_{x}, \eta_{y}\right) g(u, V)+$

$$
\begin{gathered}
a_{1}^{\prime} g\left(\eta_{x}, V\right) g\left(u, \eta_{y}\right)+a_{1}^{\prime} g\left(\eta_{y}, V\right) g\left(u, \eta_{x}\right)+ \\
\left(a_{2}^{\prime}+\frac{b_{2}}{2}\right)\left(g\left(\eta_{x}, H\right) g\left(u, \eta_{y}\right)+g\left(\eta_{y}, H\right) g\left(u, \eta_{x}\right)\right)+
\end{gathered}
$$$$
g\left(u, \eta_{x}\right) g\left(u, \eta_{y}\right) g\left(u, b_{1}^{\prime} V+2 b_{2}^{\prime} H\right) .
$$

$$
\begin{aligned}
& G\left(\widetilde{\nabla}_{\partial_{x}}\left(H^{h}+V^{v}\right), \partial_{y}\right)=G\left(-\widetilde{A}_{H^{h}+V^{v}} \partial_{x}, \partial_{y}\right)= \\
& G\left(h\left\{\mathbf{C}\left(u, H, \eta_{x}\right)\right\}+v\left\{\mathbf{D}\left(u, H, \eta_{x}\right)\right\}+h\left\{\mathbf{E}\left(u, \eta_{x}, V\right)\right\}+v\left\{\mathbf{F}\left(u, \eta_{x}, V\right)\right\}, \eta_{y}^{v}\right)= \\
& \left(b_{1}-a_{1}^{\prime}\right) g\left(\eta_{x}, V\right) g\left(u, \eta_{y}\right)+a_{1}^{\prime} g\left(\eta_{y}, V\right) g\left(u, \eta_{x}\right)+a_{1}^{\prime} g\left(\eta_{x}, \eta_{y}\right) g(u, V)+ \\
& \left(a_{2}^{\prime}-\frac{b_{2}}{2}\right)\left(g\left(\eta_{y}, H\right) g\left(u, \eta_{x}\right)-g\left(\eta_{x}, H\right) g\left(u, \eta_{y}\right)\right)+ \\
& \quad b_{1}^{\prime} g\left(u, \eta_{x}\right) g\left(u, \eta_{y}\right) g(u, V) .
\end{aligned}
$$

In virtue of the equality (3.1) the above two equations yield

$$
g\left(u, \eta_{x}\right) g\left(\eta_{y}, T\right)-g\left(u, \eta_{y}\right) g\left(\eta_{x}, T\right)=0,
$$

where $T=\left(b_{1}^{\prime}-2 a_{1}^{\prime}\right) V+\left(b_{2}^{\prime}-2 a_{2}^{\prime}\right) H$.

\section{Equation 2}

$$
\begin{gathered}
G\left(\widetilde{\nabla}_{\partial_{x}} \partial_{a}, H^{h}+V^{v}\right)=G\left(\widetilde{H}\left(\partial_{x}, \partial_{a}\right), H^{h}+V^{v}\right)= \\
G\left(\widetilde{\nabla}_{\eta_{x}^{v}}\left(\delta_{a}^{h}+M_{a}^{v}+N_{a}^{v}\right), H^{h}+V^{v}\right)= \\
G\left(h\left\{\mathbf{C}\left(u, \delta_{a}, \eta_{x}\right)\right\}+v\left\{\mathbf{D}\left(u, \delta_{a}, \eta_{x}\right)\right\}, H^{h}+V^{v}\right)+ \\
G\left(h\left\{\mathbf{E}\left(u, \eta_{x}, M_{a}+N_{a}\right)\right\}+v\left\{\mathbf{F}\left(u, \eta_{x}, M_{a}+N_{a}\right)\right\}, H^{h}+V^{v}\right)= \\
-\frac{1}{2} a_{1} R\left(H, \delta_{a}, u, \eta_{x}\right)+A^{\prime} g\left(H, \delta_{a}\right) g\left(u, \eta_{x}\right)+\left(a_{2}^{\prime}-\frac{b_{2}}{2}\right) g\left(V, \delta_{a}\right) g\left(u, \eta_{x}\right)+ \\
\left(b_{1}-a_{1}^{\prime}\right) g(u, V) g\left(N_{a}, \eta_{x}\right)+b_{2} g(u, H) g\left(N_{a}, \eta_{x}\right)+ \\
a_{1}^{\prime} g\left(u, \eta_{x}\right) g\left(V, M_{a}+N_{a}\right)+a_{1}^{\prime} g\left(u, N_{a}\right) g\left(V, \eta_{x}\right)+ \\
\left(a_{2}^{\prime}+\frac{b_{2}}{2}\right)\left[g\left(H, M_{a}+N_{a}\right) g\left(u, \eta_{x}\right)+g\left(H, \eta_{x}\right) g\left(u, N_{a}\right)\right]+ \\
g\left(u, b_{1}^{\prime} V+2 b_{2}^{\prime} H\right) g\left(u, \eta_{x}\right) g\left(u, N_{a}\right) . \quad \text { (3.3) }
\end{gathered}
$$

$$
\begin{aligned}
& G\left(\widetilde{\nabla}_{\partial_{x}}\left(H^{h}+V^{v}\right), \partial_{a}\right)=G\left(-\widetilde{A}_{H^{h}+V^{v}} \partial_{x}, \partial_{a}\right)= \\
& G\left(\widetilde{\nabla}_{\partial_{x}}\left(H^{h}+V^{v}\right), \delta_{a}^{h}+M_{a}^{v}+N_{a}^{v}\right)= \\
& G\left(h\left\{\mathbf{C}\left(u, H, \eta_{x}\right)\right\}+v\left\{\mathbf{D}\left(u, H, \eta_{x}\right)\right\}, \delta_{a}^{h}+M_{a}^{v}+N_{a}^{v}\right)+ \\
& G\left(h\left\{\mathbf{E}\left(u, \eta_{x}, V\right)\right\}+v\left\{\mathbf{F}\left(u, \eta_{x}, V\right)\right\}, \delta_{a}^{h}+M_{a}^{v}+N_{a}^{v}\right)=
\end{aligned}
$$




$$
\begin{gathered}
\frac{1}{2} a_{1} R\left(H, \delta_{a}, u, \eta_{x}\right)+\left(b_{1}-a_{1}^{\prime}\right) g\left(u, N_{a}\right) g\left(V, \eta_{x}\right)+a_{1}^{\prime} g\left(u, \eta_{x}\right) g\left(V, M_{a}+N_{a}\right)+ \\
a_{1}^{\prime} g(u, V) g\left(N_{a}, \eta_{x}\right)+\left(a_{2}^{\prime}+\frac{b_{2}}{2}\right) g\left(u, \eta_{x}\right) g\left(V, \delta_{a}\right)+ \\
\left(a_{2}^{\prime}-\frac{b_{2}}{2}\right)\left[g\left(H, M_{a}+N_{a}\right) g\left(u, \eta_{x}\right)-g\left(H, \eta_{x}\right) g\left(u, N_{a}\right)\right]+ \\
A^{\prime} g\left(H, \delta_{a}\right) g\left(u, \eta_{x}\right)+b_{1}^{\prime} g\left(u, \eta_{x}\right) g\left(u, N_{a}\right) g(u, V) .
\end{gathered}
$$

Hence, in virtue of (3.1), we get

$$
\begin{aligned}
& g\left(u, b_{1} V+b_{2} H\right) g\left(N_{a}, \eta_{x}\right)+2 g\left(u, \eta_{x}\right) g\left(A^{\prime} H+a_{2}^{\prime} V, \delta_{a}\right)+ \\
& 2 g\left(u, \eta_{x}\right) g\left(a_{1}^{\prime} V+a_{2}^{\prime} H, M_{a}+N_{a}\right)+ \\
& \quad g\left(u, N_{a}\right)\left[g\left(b_{1} V+b_{2} H, \eta_{x}\right)+2 g\left(u, \eta_{x}\right) g\left(b_{1}^{\prime} V+b_{2}^{\prime} H, u\right)\right]=0 .
\end{aligned}
$$

\section{Equation 3}

$$
\begin{gathered}
G\left(\widetilde{\nabla}_{\partial_{a}} \partial_{x}, H^{h}+V^{v}\right)=G\left(\widetilde{H}\left(\partial_{a}, \partial_{x}\right), H^{h}+V^{v}\right)= \\
G\left(\widetilde{\nabla}_{\left(\delta_{a}^{h}+M_{a}^{v}+N_{a}^{v}\right)}\left(\eta_{x}\right)^{v}, H^{h}+V^{v}\right)= \\
G\left(\left(\nabla_{\delta_{a}} \eta_{x}\right)^{v}+h\left\{\mathbf{C}\left(u, \delta_{a}, \eta_{x}\right)\right\}+v\left\{\mathbf{D}\left(u, \delta_{a}, \eta_{x}\right)\right\}, H^{h}+V^{v}\right)+ \\
G\left(h\left\{\mathbf{E}\left(u, \eta_{x}, M_{a}+N_{a}\right)\right\}+v\left\{\mathbf{F}\left(u, \eta_{x}, M_{a}+N_{a}\right)\right\}, H^{h}+V^{v}\right) .
\end{gathered}
$$

Since $\widetilde{H}\left(\partial_{a}, \partial_{x}\right)$ is symmetric, comparing the last equation with (3.3), we obtain

$$
G\left(\left(\nabla_{\delta_{a}} \eta_{x}\right)^{v}, H^{h}+V^{v}\right)=0
$$

$$
\begin{gathered}
G\left(\widetilde{\nabla}_{\partial_{a}}\left(H^{h}+V^{v}\right), \partial_{x}\right)=G\left(-\widetilde{A}_{H^{h}+V^{v}} \partial_{a}, \partial_{x}\right)= \\
G\left(\widetilde{\nabla}_{\left(\delta_{a}^{h}+M_{a}^{v}+N_{a}^{v}\right)}\left(H^{h}+V^{v}\right), \eta_{x}^{v}\right)= \\
G\left(\left(\nabla_{\delta_{a}} H\right)^{h}+h\left\{\mathbf{A}\left(u, \delta_{a}, H\right)\right\}+v\left\{\mathbf{B}\left(u, \delta_{a}, H\right)\right\}, \eta_{x}^{v}\right)+ \\
G\left(\left(\nabla_{\delta_{a}} V\right)^{v}+h\left\{\mathbf{C}\left(u, \delta_{a}, V\right)\right\}+v\left\{\mathbf{D}\left(u, \delta_{a}, V\right)\right\}, \eta_{x}^{v}\right)+ \\
G\left(h\left\{\mathbf{C}\left(u, H, M_{a}+N_{a}\right)\right\}+v\left\{\mathbf{D}\left(u, H, M_{a}+N_{a}\right)\right\}, \eta_{x}^{v}\right)+ \\
G\left(h\left\{\mathbf{E}\left(u, M_{a}+N_{a}, V\right)\right\}+v\left\{\mathbf{F}\left(u, M_{a}+N_{a}, V\right)\right\}, \eta_{x}^{v}\right)=
\end{gathered}
$$

$\frac{1}{2} a_{1} R\left(H, \delta_{a}, u, \eta_{x}\right)+a_{1} g\left(\eta_{x}, \nabla_{\delta_{a}} V\right)+b_{1} g\left(u, \eta_{x}\right) g\left(u, \nabla_{\delta_{a}} V\right)+$

$a_{2} g\left(\eta_{x}, \nabla_{\delta_{a}} H\right)+b_{2} g\left(u, \eta_{x}\right) g\left(u, \nabla_{\delta_{a}} H\right)-A^{\prime} g\left(u, \eta_{x}\right) g\left(H, \delta_{a}\right)+$

$\left(b_{1}-a_{1}^{\prime}\right) g\left(u, \eta_{x}\right) g\left(V, M_{a}+N_{a}\right)+a_{1}^{\prime} g\left(u, N_{a}\right) g\left(V, \eta_{x}\right)+a_{1}^{\prime} g(u, V) g\left(N_{a}, \eta_{x}\right)+$

$\left(a_{2}^{\prime}-\frac{b_{2}}{2}\right)\left[g\left(H, \eta_{x}\right) g\left(u, N_{a}\right)-g\left(H, M_{a}+N_{a}\right) g\left(u, \eta_{x}\right)-g\left(u, \eta_{x}\right) g\left(V, \delta_{a}\right)\right]+$

$b_{1}^{\prime} g(u, V) g\left(u, N_{a}\right) g\left(u, \eta_{x}\right)$.

Equation 4 Body Math

$$
\begin{gathered}
G\left(\widetilde{\nabla}_{\partial_{a}} \partial_{b}, H^{h}+V^{v}\right)=G\left(\widetilde{H}\left(\partial_{a}, \partial_{b}\right), H^{h}+V^{v}\right)= \\
G\left(\widetilde{\nabla}_{\left(\delta_{a}^{h}+M_{a}^{v}+N_{a}^{v}\right)}\left(\delta_{b}^{h}+M_{b}^{v}+N_{b}^{v}\right), H^{h}+V^{v}\right)= \\
G\left(\left(\nabla_{\delta_{a}} \delta_{b}\right)^{h}+h\left\{\mathbf{A}\left(u, \delta_{a}, \delta_{b}\right)\right\}+v\left\{\mathbf{B}\left(u, \delta_{a}, \delta_{b}\right)\right\}+\right. \\
\left(\nabla_{\delta_{a}}\left(M_{b}+N_{b}\right)\right)^{v}+h\left\{\mathbf{C}\left(u, \delta_{a}, M_{b}+N_{b}\right)\right\}+v\left\{\mathbf{D}\left(u, \delta_{a}, M_{b}+N_{b}\right)\right\}+ \\
h\left\{\mathbf{C}\left(u, \delta_{b}, M_{a}+N_{a}\right)\right\}+v\left\{\mathbf{D}\left(u, \delta_{b}, M_{a}+N_{a}\right)\right\}+ \\
\left.h\left\{\mathbf{E}\left(u, M_{a}+N_{a}, M_{b}+N_{b}\right)\right\}+v\left\{\mathbf{F}\left(u, M_{a}+N_{a}, M_{b}+N_{b}\right)\right\}, H^{h}+V^{v}\right)=
\end{gathered}
$$


$-a_{2} R\left(H, \delta_{b}, u, \delta_{a}\right)-$

$$
\begin{gathered}
\frac{1}{2} a_{1} R\left(H, \delta_{a}, u, M_{b}+N_{b}\right)-\frac{1}{2} a_{1} R\left(H, \delta_{b}, u, M_{a}+N_{a}\right)-\frac{1}{2} a_{1} R\left(u, V, \delta_{a}, \delta_{b}\right)+ \\
g\left(A H+a_{2} V, \nabla_{\delta_{a}} \delta_{b}\right)+g\left(B H+b_{2} V, u\right) g\left(u, \nabla_{\delta_{a}} \delta_{b}\right)+ \\
\frac{1}{2} B g(H, u)\left(g\left(M_{a}, \delta_{b}\right)+g\left(M_{b}, \delta_{a}\right)\right)+ \\
g\left(a_{1} V+a_{2} H, \nabla_{\delta_{a}}\left(M_{b}+N_{b}\right)\right)+g\left(b_{2} H+b_{1} V, u\right) g\left(u, \nabla_{\delta_{a}}\left(M_{b}+N_{b}\right)\right)+ \\
+g\left(b_{2} H+\left(b_{1}-a_{1}^{\prime}\right) V, u\right)\left(g\left(M_{a}, M_{b}\right)+g\left(N_{a}, N_{b}\right)\right)+ \\
A^{\prime}\left[g\left(H, \delta_{a}\right) g\left(u, N_{b}\right)+g\left(H, \delta_{b}\right) g\left(u, N_{a}\right)-g(V, u) g\left(\delta_{a}, \delta_{b}\right)\right]+ \\
a_{1}^{\prime} g\left(u, N_{a}\right) g\left(V, M_{b}+N_{b}\right)+a_{1}^{\prime} g\left(u, N_{b}\right) g\left(V, M_{a}+N_{a}\right)+ \\
\left(a_{2}^{\prime}-\frac{b_{2}}{2}\right)\left\{g\left(u, N_{b}\right) g\left(V, \delta_{a}\right)+g\left(u, N_{a}\right) g\left(V, \delta_{b}\right)-g(u, V)\left[g\left(M_{b}, \delta_{a}\right)+g\left(M_{a}, \delta_{b}\right)\right]\right\}+ \\
\left(a_{2}^{\prime}+\frac{b_{2}}{2}\right)\left(g\left(H, M_{a}+N_{a}\right) g\left(u, N_{b}\right)+g\left(H, M_{b}+N_{b}\right) g\left(u, N_{a}\right)\right)+ \\
g\left(u, b_{1}^{\prime} V+2 b_{2}^{\prime} H\right) g\left(u, N_{a}\right) g\left(u, N_{b}\right) .
\end{gathered}
$$

Body Math

$G\left(\widetilde{\nabla}_{\partial_{a}}\left(H^{h}+V^{v}\right), \partial_{b}\right)=G\left(-\widetilde{A}_{H^{h}+V^{v}} \partial_{a}, \partial_{b}\right)=$ $G\left(\widetilde{\nabla}_{\left(\delta_{a}^{h}+M_{a}^{v}+N_{a}^{v}\right)}\left(H^{h}+V^{v}\right), \eta_{b}^{v}\right)=$

$a_{2} R\left(H, \delta_{b}, u, \delta_{a}\right)+$

$$
\begin{gathered}
\frac{1}{2} a_{1} R\left(H, \delta_{a}, u, M_{b}+N_{b}\right)+\frac{1}{2} a_{1} R\left(H, \delta_{b}, u, M_{a}+N_{a}\right)+\frac{1}{2} a_{1} R\left(u, V, \delta_{a}, \delta_{b}\right)+ \\
A g\left(\delta_{b}, \nabla_{\delta_{a}} H\right)+a_{2} g\left(\delta_{b}, \nabla_{\delta_{a}} V\right)+a_{2} g\left(M_{b}+N_{b}, \nabla_{\delta_{a}} H\right)+a_{1} g\left(M_{b}+N_{b}, \nabla_{\delta_{a}} V\right)+ \\
\frac{1}{2} B g(H, u)\left(g\left(M_{a}, \delta_{b}\right)-g\left(M_{b}, \delta_{a}\right)\right)+ \\
g\left(u, N_{b}\right)\left(b_{2} g\left(u, \nabla_{\delta_{a}} H\right)+b_{1} g\left(u, \nabla_{\delta_{a}} V\right)\right)+ \\
A^{\prime}\left(-g\left(H, \delta_{a}\right) g\left(u, N_{b}\right)+g\left(H, \delta_{b}\right) g\left(u, N_{a}\right)+g(V, u) g\left(\delta_{a}, \delta_{b}\right)\right)+ \\
\left(b_{1}-a_{1}^{\prime}\right) g\left(u, N_{b}\right) g\left(V, M_{a}+N_{a}\right)+a_{1}^{\prime} g\left(u, N_{a}\right) g\left(V, M_{b}+N_{b}\right)+
\end{gathered}
$$

$a_{1}^{\prime} g(V, u)\left[g\left(M_{a}, M_{b}\right)+g\left(N_{a}, N_{b}\right)\right]+$

$$
\begin{gathered}
\left(a_{2}^{\prime}-\frac{b_{2}}{2}\right)\left(g(u, V) g\left(M_{b}, \delta_{a}\right)-g\left(u, N_{b}\right) g\left(V, \delta_{a}\right)\right)+ \\
\left(a_{2}^{\prime}+\frac{b_{2}}{2}\right)\left(g(u, V) g\left(M_{a}, \delta_{b}\right)+g\left(u, N_{a}\right) g\left(V, \delta_{b}\right)\right)+ \\
\left(a_{2}^{\prime}-\frac{b_{2}}{2}\right)\left[g\left(-H, M_{a}+N_{a}\right) g\left(u, N_{b}\right)+g\left(H, M_{b}+N_{b}\right) g\left(u, N_{a}\right)\right]+
\end{gathered}
$$

$b_{1}^{\prime} g(u, V) g\left(u, N_{a}\right) g\left(u, N_{b}\right)$.

Applying (3.1), we find

$$
\begin{gathered}
0=g\left(A H+a_{2} V, \nabla_{\delta_{a}} \delta_{b}\right)+ \\
g\left(B H+b_{2} V, u\right) g\left(u, \nabla_{\delta_{a}} \delta_{b}\right)+g\left(a_{2} H+a_{1} V, \nabla_{\delta_{a}}\left(M_{b}+N_{b}\right)\right)+ \\
a_{2} g\left(M_{b}+N_{b}, \nabla_{\delta_{a}} H\right)+a_{1} g\left(M_{b}+N_{b}, \nabla_{\delta_{a}} V\right)+ \\
A g\left(\delta_{b}, \nabla_{\delta_{a}} H\right)+a_{2} g\left(\delta_{b}, \nabla_{\delta_{a}} V\right)+g\left(B H+b_{2} V, u\right) g\left(M_{a}, \delta_{b}\right)+ \\
g\left(u, b_{2} H+b_{1} V\right) g\left(u, \nabla_{\delta_{a}}\left(M_{b}+N_{b}\right)+\right. \\
g\left(u, N_{b}\right)\left[b_{1} g\left(u, \nabla_{\delta_{a}} V\right)+b_{2} g\left(u, \nabla_{\delta_{a}} H\right)+g\left(b_{2} H+b_{1} V, M_{a}+N_{a}\right)\right]+ \\
g\left(u, b_{2} H+b_{1} V\right)\left[g\left(M_{a}, M_{b}\right)+g\left(N_{a}, N_{b}\right)\right]+2 g\left(u, N_{a}\right) \times \\
{\left[g\left(A^{\prime} H+a_{2}^{\prime} V, \delta_{b}\right)+g\left(a_{2}^{\prime} H+a_{1}^{\prime} V, M_{b}+N_{b}\right)+g\left(u, N_{b}\right) g\left(b_{2}^{\prime} H+b_{1}^{\prime} V, u\right)\right] .}
\end{gathered}
$$




\section{Main results}

The first proposition of this section establishes a number of various relations that allow us to show that the right hand sides of the pairs of equations in each subsection of the former section satisfy (3.1). The results are presented in Proposition 4.1. Theorem 4.1 states the condition sufficient for the space normal to $L M$ being spanned by lifts of vectors tangent to $M$. The main results are presented in Theorems 4.2 and 4.3.

Proposition 4.1. Let $\widetilde{f}$ be the immersion given by (2.3) defined by the isometric immersion $f: M \rightarrow(N, g)$ into a Riemannian manifold. Suppose, moreover, that $T N$ is endowed with non-degenerate g-natural metric $G$. Then in the notation as above the following identities are satisfied.

1 .

$$
g\left(\eta_{x}, S\right)=0
$$

where $S=a_{2} H+a_{1} V+g\left(u, b_{2} H+b_{1} V\right) u$.

2.

$$
g(u, S)=g\left(N_{a}, S\right)=0 .
$$

3.

$$
g\left(\nabla_{\delta_{a}} \eta_{x}, S\right)=0
$$

4.

$$
g\left(\eta_{x}, \nabla_{\delta_{a}} S\right)=0
$$

5.

$$
g\left(u, \nabla_{\delta_{a}} S\right)=g\left(N_{b}, \nabla_{\delta_{a}} S\right)=0
$$

6.

$$
g\left(\nabla_{\delta_{a}} u, S\right)=g\left(\nabla_{\delta_{a}} N_{b}, S\right)=0 .
$$

7.

$$
g\left(\delta_{a}, A H+a_{2} V\right)=g\left(M_{a}, a_{2} H+a_{1} V\right)=0 .
$$

8.

$$
g\left(\delta_{a}, A^{\prime} H+a_{2}^{\prime} V\right)=g\left(M_{a}, a_{2}^{\prime} H+a_{1}^{\prime} V\right)=0 .
$$

9.

$$
g\left(M_{b}, M_{a}\right)+g\left(u, \nabla_{\delta_{a}} M_{b}\right)=0 .
$$

10.

$$
g\left(M_{b}, \delta_{a}\right)+g\left(u, \nabla_{\delta_{b}} \delta_{a}\right)=0 .
$$

11.

$$
g\left(\nabla_{\delta_{a}} \eta_{x}, \delta_{b}\right)+g\left(\eta_{x}, \nabla_{\delta_{a}} \delta_{b}\right)=0 .
$$

Moreover, if $M$ is not a hypersurface of $N$, then

12.

$$
X_{u}=g\left(u, b_{2} H+b_{1} V\right)=0 .
$$

13.

$$
X_{\eta_{x}}=g\left(\eta_{x}, b_{2} H+b_{1} V\right)=0 .
$$

14.

$$
Y_{\eta_{x}}=g\left(\eta_{x}, b_{2}^{\prime} H+b_{1}^{\prime} V\right)=0 .
$$

15.

$$
Y_{u}=g\left(u, b_{2}^{\prime} H+b_{1}^{\prime} V\right)=0 .
$$


16.

$$
Z_{\eta_{x}}=g\left(\eta_{x}, a_{2}^{\prime} H+a_{1}^{\prime} V\right)=0, \quad Z_{u}=g\left(u, a_{2}^{\prime} H+a_{1}^{\prime} V\right)=0 .
$$

17.

$$
g\left(\eta_{x}, a_{2} H+a_{1} V\right)=0
$$

Finaly

18.

$$
S=a_{2} H+a_{1} V .
$$

Proof. (4.1) results from

$$
G\left(\partial_{x}, H^{h}+V^{v}\right)=G\left(\eta_{x}^{v}, H^{h}+V^{v}\right)=0 .
$$

Then (4.2) is obvious since $u=v^{y} N_{y}^{r} \partial_{r}=v^{y} \eta_{y}$ and $N_{a}=N_{a}^{y} \eta_{y}$ are normal to $M$. Now (4.3) is a consequence of (3.5), whence, by (4.1), (4.4) results.

Once again, by orthogonality of $u$ and $N_{a}$ with respect to $M$, we have (4.5). Consequently, in virtue of (4.2), we obtain (4.6).

Observe that the identity

$$
(3.4)-(3.6)+g\left(\eta_{x}, \nabla_{\delta_{a}} S\right)-\sum_{y} \frac{\partial}{\partial v^{y}} g\left(\eta_{x}, S\right) N_{a}^{y}=0
$$

gives

On the other hand, relations

$$
g\left(\delta_{a}, A^{\prime} H+a_{2}^{\prime} V\right)+g\left(M_{a}, a_{2}^{\prime} H+a_{1}^{\prime} V\right)=0 .
$$

$$
G\left(\partial_{a}, H^{h}+V^{v}\right)=G\left(\delta_{a}^{h}+M_{a}^{v}+N_{a}^{v}, H^{h}+V^{v}\right)=0
$$

and (4.2) yield

$$
g\left(\delta_{a}, A H+a_{2} V\right)+g\left(M_{a}, a_{2} H+a_{1} V\right)=0 .
$$

Differentiating (4.19) with respect to $v^{x}$ and using (4.18) we find

$$
g\left(M_{a x}, a_{2} H+a_{1} V\right)=0,
$$

where $M_{a x}=\nabla_{\delta_{a}} N_{x}^{r} \partial_{r}$. Consequently, (4.19) yields (4.7). Hence, by differentiating with respect to $v^{x},(4.8)$ results.

Since $M_{a}, \delta_{a}$ are tangent to $M$ and $u, \eta_{x}$ are normal, by covariant differentiation of $g\left(u, M_{a}\right)=0, g\left(u, \delta_{a}\right)=0$, $g\left(\eta_{x}, \delta_{a}\right)=0$ we get $(4.9)$ - (4.11).

Differentiating (4.1) with respect to $v^{y}$ we get

$$
g\left(\eta_{x}, \eta_{y}\right) X_{u}+2 g\left(\eta_{x}, u\right) g\left(u, \eta_{y}\right) Y_{u}+g\left(\eta_{x}, u\right) X_{\eta_{y}}+2 g\left(u, \eta_{y}\right) Z_{\eta_{x}}=0,
$$

where $X_{u}=g\left(u, b_{2} H+b_{1} V\right), X_{\eta_{x}}=g\left(\eta_{x}, b_{2} H+b_{1} V\right), Y_{u}=g\left(u, b_{2}^{\prime} H+b_{1}^{\prime} V\right), Z_{u}=g\left(u, a_{2}^{\prime} H+a_{1}^{\prime} V\right)$ and $Z_{\eta_{x}}=$ $g\left(\eta_{x}, a_{2}^{\prime} H+a_{1}^{\prime} V\right)$.

Transvecting in turn with $v^{x}, v^{y}, v^{x} v^{y}$ and, finally, contracting wit $g^{x y}$ we get for each $x=m+1, \ldots, n$ a system of four equations:

$$
\begin{aligned}
v_{x} X_{u}+2 r^{2} v_{x} Y_{u}+r^{2} X_{\eta_{x}}+2 v_{x} Z_{u} & =0, \\
v_{x} X_{u}+2 r^{2} v_{x} Y_{u}+v_{x} X_{u}+2 r^{2} Z_{\eta_{x}} & =0, \\
r^{2}\left(X_{u}+r^{2} Y_{u}+Z_{u}\right) & =0, \\
(n-m+1) X_{u}+2 r^{2} Y_{u}+2 Z_{u} & =0,
\end{aligned}
$$

where $v_{x}=g\left(u, \eta_{x}\right)$. Solving it with respect to $X, X_{\eta_{x}}, Y, Z_{\eta_{x}}$ we obtain

$$
X_{u}=X_{\eta_{x}}=0, \quad Y_{u}=-\frac{Z_{u}}{r^{2}}, \quad Z_{\eta_{x}}=-\frac{v_{x} Z_{u}}{r^{2}}
$$

for any $u=v^{x} \eta_{x} \neq 0$. By continuity, $X_{u}=X_{\eta_{x}}=0$ hold for any $u$. Then $\frac{\partial}{\partial v^{z}} X_{\eta_{x}}=Y_{\eta_{x}} g\left(\eta_{z}, u\right)=0$ for all $u \neq 0$, whence, in virtue of continuity, $Y_{\eta_{x}}=0$ for any $u$. Consequently, we have $Y_{u}=0$. Now, $Z_{u}=0$ follows from (4.20) and $Z_{\eta_{x}}=0$ results from (4.21). Finally, (4.17) is a consequence of (4.1) and (4.12). Thus the lemma is proved. 
Theorem 4.1. Let $(x, u)$ be a point of $L M$ immersed in $T N$. If codim $M>1$ and

$$
a_{2} b_{1}-a_{1} b_{2} \neq 0
$$

at $t=g(u, u)$, then the normal space at $(x, u) \in L M$ is spanned by lifts of vectors tangent to $M$.

Proof. It results from the identities (4.17) and (4.13). Note that other conditions, similar to that of (4.22) can be deduced in the same way from (4.17), (4.16), (4.13) and (4.14).

We shall prove that the conditions $\operatorname{codim} M>1$ and (4.22) are essential in that sense that there exist immersion $f: M \longrightarrow N, M$ being a hypersurface of $N$, and a metric $G$ on $T N$ satisfying $a_{2} b_{1}-a_{1} b_{2}=0$ such that the normal component of at least one of the vectors $H, V$ does not vanish.

Example 4.1. Let $f: S^{1} \longrightarrow\left(R^{2}\right.$, Euclid metric) be the immersion given by $f(t)=[\cos t$, sin $t]$. The vector tangent to $S^{1}$ is $\mathbf{s}=[-\sin t, \cos t]$ and the normal one is $\mathbf{n}=[\cos t, \sin t]$. Then

$$
\mathbf{s}^{v}=[0,0,-\sin t, \cos t], \quad \mathbf{s}^{h}=[-\sin t, \cos t, 0,0],
$$

$$
\mathbf{n}^{v}=[0,0, \cos t, \sin t], \quad \mathbf{n}^{h}=[\cos t, \sin t, 0,0]
$$

The vectors tangent to $L\left(S^{1}\right)$ are

Suppose

$$
\frac{\partial}{\partial t}=\mathbf{s}^{h}+v \mathbf{s}^{v}, \quad \frac{\partial}{\partial v}=\mathbf{n}^{v}
$$

$$
H^{h}+V^{v}=\alpha \mathbf{s}^{h}+\beta \mathbf{n}^{h}+\gamma \mathbf{s}^{v}+\delta \mathbf{n}^{v}
$$

and consider a non-degenerate $g$-natural metric on $T R^{2}$ such that $B=b_{1}=b_{2}=0$.

Then

and

$$
G\left(\frac{\partial}{\partial v}, H^{h}+V^{v}\right)=G\left(\mathbf{n}^{v}, H^{h}+V^{v}\right)=a_{2} g(\mathbf{n}, H)+a_{1} g(\mathbf{n}, V)=a_{2} \beta+a_{1} \delta
$$

$$
G\left(\frac{\partial}{\partial t}, H^{h}+V^{v}\right)=G\left(\mathbf{s}^{h}, H^{h}+V^{v}\right)=A g(\mathbf{s}, H)+a_{2} g(\mathbf{s}, V)=A \alpha+a_{2} \gamma
$$

We put

$$
\alpha=-\frac{a_{2}+v a_{1}}{A+v a_{2}} \gamma \neq 0, \quad \beta=-\frac{a_{1}}{a_{2}} \delta \neq 0 .
$$

The restriction on codimension can be omitted as the next proposition shows.

Proposition 4.2. If $a_{2}=b_{2}=0$ for all $\left.t \in<0, \infty\right)$, then the normal bundle of $L M$ at a point $(x, u), x \in M, u \in T_{x} M$, is spanned by the vectors $\eta_{y}^{h}$ and $\delta_{a}^{v}-\frac{a_{1}}{A}\left(\nabla_{\delta_{a}} u\right)^{h}$.

Proof. Direct calculation. Property (4.10) is applied.

Applying Proposition 4.1 to (3.2), (3.3) and (3.7) we obtain

Theorem 4.2. Let $M$, codim $M>1$, be a submanifold isometrically immersed in a manifold $N$. Then along LM we have:

1.

$$
\begin{aligned}
\widetilde{G}\left[H^{h}+V^{v}, \widetilde{\nabla}_{\partial_{x}} \partial_{y}\right]=\widetilde{G}\left[H^{h}+V^{v}, \widetilde{H}\left(\partial_{x}, \partial_{y}\right)\right]= \\
\quad g\left[H, \frac{b_{2}}{2} g\left(u, \eta_{x}\right) \eta_{y}+\frac{b_{2}}{2} g\left(u, \eta_{y}\right) \eta_{x}+a_{2}^{\prime} g\left(\eta_{x}, \eta_{y}\right) u+b_{2}^{\prime} g\left(u, \eta_{x}\right) g\left(u, \eta_{y}\right) u\right]
\end{aligned}
$$

2.

$$
\begin{aligned}
& \widetilde{G}\left[H^{h}+V^{v}, \widetilde{\nabla}_{\partial_{x}} \partial_{b}\right]=\widetilde{G}\left[H^{h}+V^{v}, \widetilde{H}\left(\partial_{x}, \partial_{a}\right)\right]= \\
& g\left[H, \frac{1}{2} a_{1} R\left(u, \eta_{x}\right) \delta_{a}\right]+ \\
& g\left[H, \frac{b_{2}}{2} g\left(u, N_{a}\right) \eta_{x}+\frac{b_{2}}{2} g\left(u, \eta_{x}\right)\left(M_{a}+N_{a}\right)+a_{2}^{\prime} g\left(N_{a}, \eta_{x}\right) u+b_{2}^{\prime} g\left(u, \eta_{x}\right) g\left(u, N_{a}\right) u\right]- \\
& g\left[V, \frac{b_{2}}{2} g\left(u, \eta_{x}\right) \delta_{a}\right],
\end{aligned}
$$


3.

$$
\begin{gathered}
\widetilde{G}\left[H^{h}+V^{v}, \widetilde{\nabla}_{\partial_{a}} \partial_{b}\right]=\widetilde{G}\left[H^{h}+V^{v}, \widetilde{H}\left(\partial_{a}, \partial_{b}\right)\right]= \\
g\left[A H+a_{2} V, \nabla_{\delta_{a}} \delta_{b}\right]-a_{2} R\left(H, \delta_{b}, u, \delta_{a}\right)- \\
\frac{a_{1}}{2}\left[R\left(H, \delta_{a}, u, M_{b}+N_{b}\right)+R\left(H, \delta_{b}, u, M_{a}+N_{a}\right)+R\left(u, V, \delta_{a}, \delta_{b}\right]+\right. \\
g\left[a_{2} H+a_{1} V, \nabla_{\delta_{a}}\left(M_{b}+N_{b}\right)\right]- \\
g(u, V)\left[A^{\prime} g\left(\delta_{a}, \delta_{b}\right)+a_{1}^{\prime}\left(g\left(M_{a}, M_{b}\right)+g\left(N_{a}, N_{b}\right)\right)+2 a_{2} g\left(M_{a}, \delta_{b}\right)\right]- \\
\frac{1}{2} b_{2}\left[g\left(u, N_{a}\right) g\left(V, \delta_{b}\right)+g\left(u, N_{b}\right) g\left(V, \delta_{a}\right)\right]+ \\
g\left[H, \frac{b_{2}}{2} g\left(u, N_{a}\right)\left(M_{b}+N_{b}\right)+\frac{b_{2}}{2} g\left(u, N_{b}\right)\left(M_{a}+N_{a}\right)\right]+ \\
b_{2}^{\prime} g(u, H) g\left(u, N_{a}\right) g\left(u, N_{b}\right)
\end{gathered}
$$

for a nondegenerate g-natural metric $\widetilde{G}$.

Proof. Straightforward computation.

Remark 4.1. The third equation of the last Proposition can be written as

$$
\begin{gathered}
\widetilde{G}\left[H^{h}+V^{v}, \widetilde{\nabla}_{\partial_{a}} \partial_{b}\right]=\widetilde{G}\left[H^{h}+V^{v}, \widetilde{H}\left(\partial_{a}, \partial_{b}\right)\right]= \\
G\left[H^{h}+V^{v},\left(\nabla_{\delta_{a}} \delta_{b}\right)^{h}+\left(\nabla_{\delta_{a}} \nabla_{\delta_{b}} u\right)^{v}\right]-g\left(B H+b_{2} V, u\right) g\left(u, \nabla_{\delta_{a}} \nabla_{\delta_{b}} u\right)- \\
a_{2} R\left(H, \delta_{b}, u, \delta_{a}\right)-\frac{a_{1}}{2}\left[R\left(H, \delta_{a}, u, M_{b}+N_{b}\right)+R\left(H, \delta_{b}, u, M_{a}+N_{a}\right)+R\left(u, V, \delta_{a}, \delta_{b}\right]+\right. \\
g(u, V)\left[A^{\prime} g\left(\delta_{a}, \delta_{b}\right)+a_{1}^{\prime}\left(g\left(M_{a}, M_{b}\right)+g\left(N_{a}, N_{b}\right)\right)+2 a_{2} g\left(M_{a}, \delta_{b}\right)\right]- \\
\frac{1}{2} b_{2}\left[g\left(u, N_{a}\right) g\left(V, \delta_{b}\right)+g\left(u, N_{b}\right) g\left(V, \delta_{a}\right)\right]+ \\
g\left[H, \frac{b_{2}}{2} g\left(u, N_{a}\right)\left(M_{b}+N_{b}\right)+\frac{b_{2}}{2} g\left(u, N_{b}\right)\left(M_{a}+N_{a}\right)\right]+ \\
b_{2}^{\prime} g(u, H) g\left(u, N_{a}\right) g\left(u, N_{b}\right) .
\end{gathered}
$$

Definition 4.1. A distribution $D$ on a manifold $M$ is said to be totally geodesic if it is invariant with respect to covariant differentiation, i.e. $\nabla_{X} Y \in D$ for all $X, Y \in D$.

Theorem 4.3. Let $M$, codim $M>1$, be a submanifold isometrically immersed in a manifold $(N, g)$. Suppose that $L M$ is a submanifold isometrically immersed by (2.3) in $T N$ with non-degenerate g-natural metric $G$.

1. If either the normal bundle of $L M$ is spanned by vectors of the form $H^{h}+V^{v}$, where $H$ and $V$ are tangent to $M$ or $b_{2}=a_{2}^{\prime}=0$ along $M$, then vector fields $\left\{\partial_{x}\right\}, x=m+1, \ldots, n$ define on $L M$ the totally geodesic distribution that is involutive.

2. If

(a) $a_{1}=0, b_{2}=0, a_{2}=$ const $\neq 0$ along $M$ or

(b) $N$ is a space of constant curvature and $a_{2}^{\prime}=0, b_{2}=0$ along $M$,

then $L M$ is mixed totally geodesic.

Here, along $M$,

$$
\nabla_{\delta_{a}} u=\nabla_{\delta_{a}}\left(v^{y} \eta_{y}\right)=\nabla_{\delta_{a}}\left(v^{y} N_{y}^{r} \frac{\partial}{\partial x^{r}}\right)=M_{a}+N_{a}
$$

Proof. In virtue of the assumptions, the first equation of Theorem 4.2 yields $\widetilde{G}\left[H^{h}+V^{v}, \widetilde{H}\left(\partial_{x}, \partial_{y}\right)\right]=0$. Hence $\widetilde{\nabla}_{\partial_{x}} \partial_{y}$ is tangent to $L M$. Since $\partial_{x}=\eta_{x}^{v}$ are vertical vector fields, the distribution is involutive. This proves the first point. The proof of the second one is obvious. 


\section{References}

[1] Abbassi, M. T. K., Yampolsky, A., Transverse totally geodesic submanifolds of the tangent bundle. Publ. Math. Debrecen 64/1-2 (2004), 129-154.

[2] Abbassi, M. T. K., Sarih, Maâti, On natural metrics on tangent bundles of Riemannian manifolds. Arch. Math. (Brno) 41 (2005), no. 1, 71-92.

[3] Abbassi, M. T. K., Sarih, Maâti, On some hereditary properties of Riemannian $g$-natural metrics on tangent bundles of Riemannian manifolds. Differential Geom. Appl. 22 (2005), no. 1, 19-47.

[4] Abbassi, M. T. K., Métriques Naturelles Riemanniennes sur la Fibré tangent une variété Riemannienne, Editions Universitaires Européénnes, Saarbrücken, Germany, 2012.

[5] Degla, S., Ezin, J. P., Todjihounde, L., On g-natural metrics of constant sectional curvature on tangent bundles. Int. Electronic J. Geom. 2(1) (2009), p. 74-94.

[6] Deshmukh S., Al-Odan, H., Shaman, T. A., Tangent bundle of the hypersurfaces in a Euclidean space. Acta Math. Acad. Pedagog. Nyíregyháziensis 23(1) (2007),71-87.

[7] Dombrowski, P., On the Geometry of Tangent Bundle. J. Reine Angew. Math. 210 (1962), p. 73-88.

[8] Ewert-Krzemieniewski, S., On a classification of Killing vector fields on a tangent bundle with $g$-natural metric. arXiv:1305:3817v1.

[9] Ewert-Krzemieniewski, S., On a Killing vector fields on a tangent bundle with $g$-natural metric Part I. Note Mat. 34 no. 2, (2014), 107-133.

[10] Ewert-Krzemieniewski, S., Totally geodesic submanifolds in tangent bundle with $g$-natural metric. Int. J. Geom. Methods Mod. Phys. 11 (2014), no. 9, 1460033 (9 pages).

[11] Ewert-Krzemieniewski, S., On a class of submanifolds in tangent bundle with $g$-natural metric. arXiv:1411.3274.

[12] Ewert-Krzemieniewski, S., On a class of submanifolds in a tangent bundle with a $g$-natural metric. Coll. Math., 150 no.1, (2017), 121-133.

[13] Kowalski, O., Sekizawa, M., Natural transformations of Riemannian metrics on manifolds to metrics on tangent bundles, A classification. Bull. Tokyo Gakugei Univ. (4) 40 (1988), 1-29.

[14] Yano, K., Kon, M., Structures on Manifolds. World Scientific, 1984.

[15] Yano, K., Ishihara, S., Tangent and cotangent bundles. Marcel Dekker, Inc. New York, 1973.

[16] Yano, K., Submanifolds with parallel mean curvature vector. J. Diff. Geom. 6 (1971), 95-118.

\section{Affiliations}

STANISŁAW EWERT-KRZEMIENIEWSKI

AdDress: West Pomeranian University of Technology Szczecin, School of Mathematics

Al. Piastów 17, 70-310 Szczecin, Poland

E-MAIL: ewert@zut.edu.pl

ORCID ID:0000-0003-0644-6268 